\title{
VALORACIÓN DEL CONTROL DE CALIDAD DE MATERIALES EN ESTRUCTURAS DE EDIFICACIÓN EFECTUADO EN CANTABRIA, EN EL QUINQUENIO 1984-1988
}

\author{
(EVALUATION OF QUALITY CONTROL OF MATERIALS IN BUILDING STRUCTURES \\ CARRIED OUT IN CANTABRIA DURING THE FIVE-YEAR PERIOD 1984-1988)
}

Luis Villegas Cabredo, Dr. Ingeniero de Caminos

Departamento de Estructuras. Universidad de Cantabria. ESPAÑA

Fecha de recepción: 31-X-89

\section{RESUMEN}

Se recoge una integración de los datos relativos al control de calidad de materiales en estructuras de edificación suministrados por los Laboratorios que operan en la región de Cantabria durante el período 1984-88.

Con estos datos y los que proporcionan las estadísticas de construcción (número de viviendas y metros cuadrados construidos) se hace una valoración del grado de cumplimiento de la Instrucción EH-88 para estructuras de hormigón en lo referente a su Capítulo IX Control de Materiales.

Las Conclusiones del estudio muestran, en los referente al Control del Hormigón, grados de cumplimiento de la normativa del 47 por 100 en estructuras para viviendas y del 88 por 100 en edificios no residenciales, $y$ parece confirmarse un incremento progresivo de este control. Los resultados son más desfavorables en el cumplimiento del Control del Acero (15 por 100 en viviendas y 44 por 100 en no residenciales) aunque se apunta, también, una tendencia hacia la realización de mayor control a lo largo del tiempo.

\section{SUMMARY}

Compilation of data related to quality control of materials in building structures, supplied by Laboratories operating in the Cantabria region, for the period 1984-1988.

This data together with the information derived from building statistics (number of homes and square meters built) is used to evaluate the level of compliance with Instructions EH-88 for concrete structures, referred to in Chapter IX "Materials Control".

This study's conclusions show that compliance with the Concrete Control rules was $47 \%$ for homes and $88 \%$ in nonresidential buildings, which seems to confirm a progressive increase in this control. The least favorable results referred to compliance with Steel Controls $15 \%$ in homes and $44 \%$ in non-residential buildings) although, here too, there is a tendency towards a greater control during this period.

\section{TOMA DE DATOS}

En la figura 1, relativa a construcción de viviendas, se muestran los números de determinaciones de resistencias de hormigón obtenidas y de diámetros de armaduras controlados en los diferentes años del estudio. También, se recogen (Informes Seopan [3]) las viviendas terminadas anualmente en la región.

La figura 2 ofrece para la edificación no residencial (do- cente, sanitaria, administrativa, industrial y deportiva) los controles de materiales realizados en el quinquenio.

Es opinión de los tres Laboratorios que proporcionan los datos de este estudio (véase su relación en el apartado de Agradecimientos) que ellos realizan la práctica totalidad del control de estructuras que se construyen en la región, por lo que las valoraciones que se hagan seguidamente mostrarán el grado de control real que se está llevando a cabo. 


\section{CONTROL DE ESTRUCTURAS PARA VIVIENDAS}

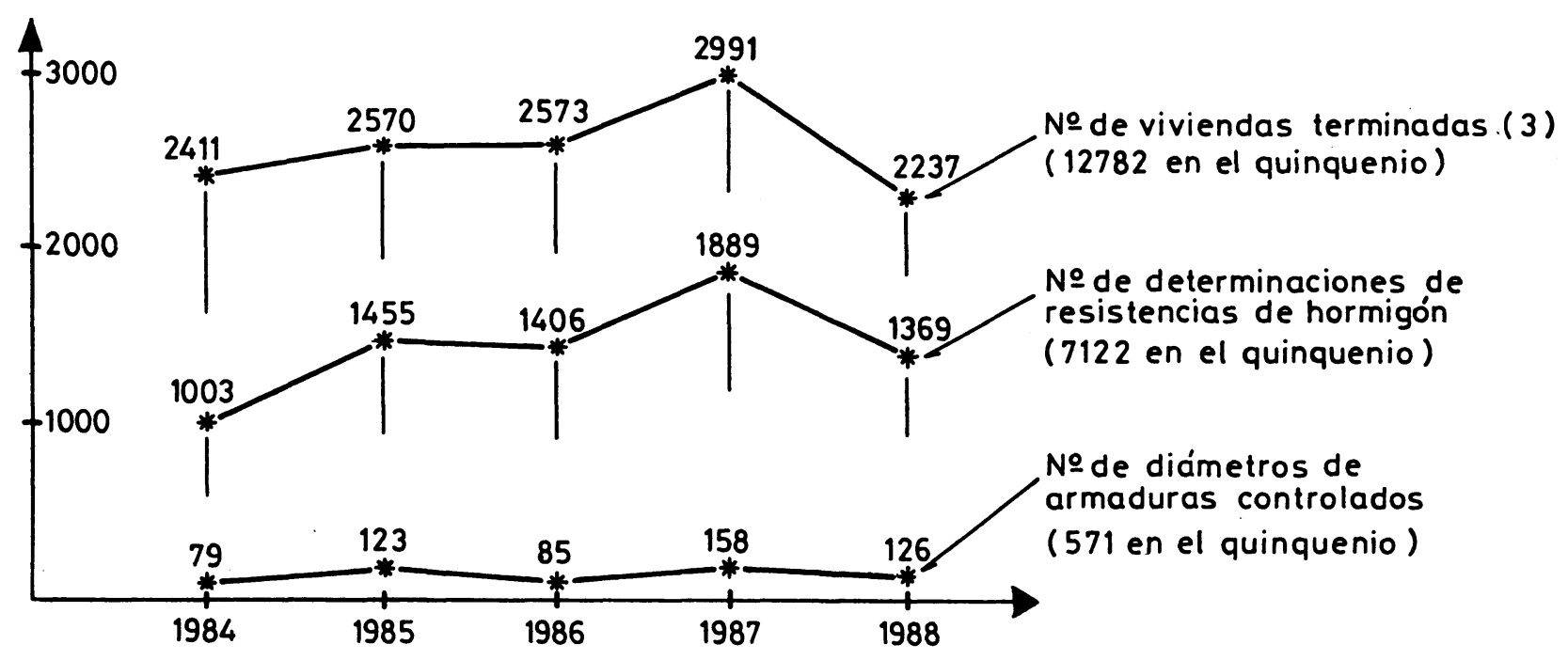

Fig. 1.-Control realizado en Cantabria en estructuras para viviendas y número de éstas en el período 1984-88.

\section{CONTROL EN EDIFICIOS NO RESIDENCIALES}

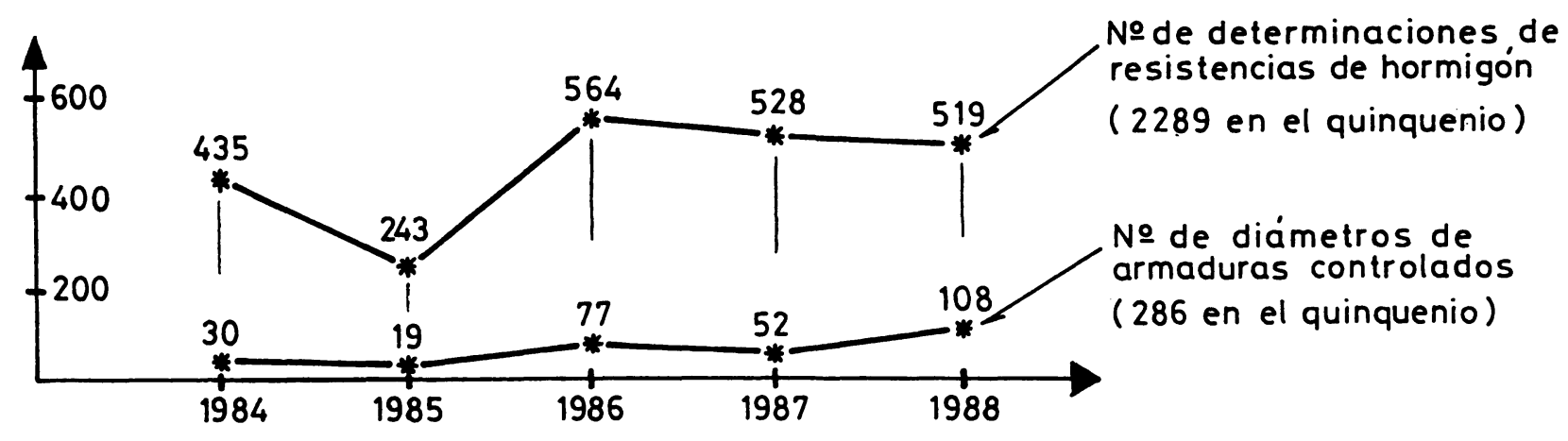

Fig. 2.-Control de materiales en estructuras de edificios no residenciales (Cantabria, 1984-88).

\section{VALORACIÓN DEL CONTROL DEL HORMIGÓN}

\subsection{Normativa}

La Instrucción EH-88 [2] establece en su Artículo 69. relativo a los Ensayos de control del hormigón la obligatoriedad en todos los casos de este tipo de ensayos. Para el caso de control a nivel normal, lo habitual en las estructuras de edificación, EH específica los criterios de formación de lotes de control (Cuadro 1).

\subsection{Control realizado en estructuras para viviendas}

En un edificio denominaremos $\mathrm{K}$ al parámetro igual al Número mínimo de determinaciones de resistencia que son necesarias por vivienda construida, para controlar adecuadamente su estructura de acuerdo con la normativa vigente. Su valor concreto depende de numerosos factores (tipo de cimentación, existencia o no de muros de sótano, superficie de las viviendas, etc.), pero puede ser del orden que refleja el Cuadro 2 en función del número de viviendas del edificio. 
CUADRO 1. CRITERIOS DE FORMACIÓN DE LOTES PARA EL CONTROL DE HORMIGÓN A NIVEL NORMAL (INSTRUCCIÓN EH-88)

\begin{tabular}{|l|c|c|c|}
\hline \multirow{2}{*}{ LIMITE SUPERIOR } & \multicolumn{3}{|c|}{ TIPO DE ELEMENTOS ESTRUCTURALES } \\
\cline { 2 - 4 } & $\begin{array}{c}\text { ELEMENTOS } \\
\text { COMPRIMIDOS } \\
\text { (PILARES, MUROS } \\
\text { PORTANTES, ETC.) }\end{array}$ & $\begin{array}{c}\text { ELEMENTOS EN } \\
\text { FLEXION SIMPLE } \\
\text { (VIGAS, FORJADOS, } \\
\text { MUROS DE } \\
\text { CONTENCION, } \\
\text { ETC.) }\end{array}$ & $\begin{array}{c}\text { MACIZOS (ZAPATAS, } \\
\text { ESTRIBOS DE } \\
\text { PUENTE, BLOQUES, } \\
\text { ETC.) }\end{array}$ \\
\hline $\begin{array}{l}\text { Volumen de hormigón } \\
\text { Número de amasadas }\end{array}$ & $50 \mathrm{~m}^{3}$ & $100 \mathrm{~m}^{3}$ & $100 \mathrm{~m}^{3}$ \\
Tiempo de hormigonado & 25 & 50 & 100 \\
Superficie construida & 2 semanas & 2 semanas & 1 semana \\
Número de plantas & $1.000 \mathrm{~m}^{2}$ & $1.000 \mathrm{~m}^{2}$ & - \\
\hline
\end{tabular}

CUADRO 2. VALORES HABITUALES DE K (NÚMERO DE DETERMINACIONES DE RESISTENCIA POR VIVIENDA) EN ESTRUCTURAS PARA DIFERENTE NÚMERO DE VIVIENDAS

\begin{tabular}{|c|c|c|c|}
\hline N. ${ }^{\circ}$ DE VIVIENDAS & Grande $(>40)$ & Medio $(\approx 20)$ & Pequeño $(<10)$ \\
\hline $\mathrm{K}$ & 0,5 a 0,7 & 0,9 a 1,3 & 2,0 a 8,0 \\
\hline
\end{tabular}

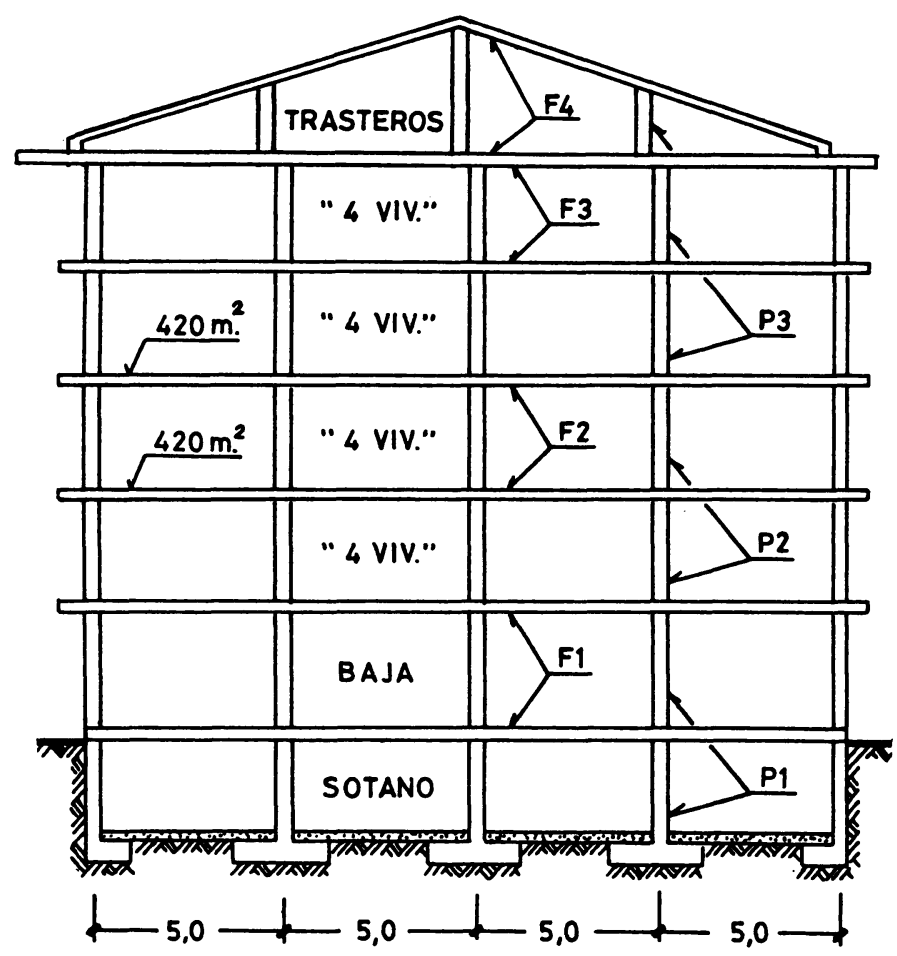

\section{CONSUMO DE MATERIALES}

\begin{tabular}{lcc|}
\hline ELEMENTO & HORMIGON $\left(\mathrm{m}^{3}\right)$ & ACERO $(t)$ \\
Forjado (PI. tipo) & 48 & 5,5 \\
Pilares (PI. tipo) & 11 & 1,3 \\
Muros & 132 & 7,4 \\
Zapatas & 41 & 1,4 \\
\hline
\end{tabular}

FORMACION DE LOTES DE CONTROL

\begin{tabular}{|lcc|}
\hline ELEMENTO & N. ${ }^{\circ}$ LOTES & N..$^{\circ}$ DETERMIN. \\
Forjados & 4 & 8 \\
Pilares & 4 & 8 \\
Muros & 3 & 6 \\
Zapatas & 1 & 2 \\
\hline
\end{tabular}

Fig. 3.-Estructura tipo para 20 viviendas. Número mínimo de determinaciones de resistencia por vivienda $K=1,2$. 
Dado que las estructuras para un número medio de viviendas (con $\mathrm{K}$ del orden de 1,0) pueden considerarse representativas del total de estas estructuras, en la medida que el gráfico que representa a lo largo del tiempo el número de determinaciones de resistencia se acerque al número de viviendas construidas (figura 1) nos encontraremos más próximos al cumplimiento de la normativa de control.

Seguidamente obtendremos la estructura tipo que se ha construido en Cantabria durante el período en estudio, que adoptaremos como representativa del mismo, y su valor de $\mathrm{K}$ le tomaremos como comparador entre el control que debiera haberse realizado y el realmente hecho.

Del total de los datos suministrados por los Laboratorios en 182 estructuras tenemos su correlación con el número de viviendas (3651) al que estaban destinadas, resultando de ello como estructura tipo una para 20 viviendas. En la figura 3 se representa una posible configuración de esta estructura, las mediciones de materiales de los diferentes elementos (se han admitido los consumos habituales) y la formación de lotes de control según las especificaciones de EH, resultando un valor de $\mathrm{K}$ igual a 1,2.

En la figura 1 se mostró el total de determinaciones de resistencia efectuado por los Laboratorios y el número de viviendas construidas durante el quinquenio en estudio, resultando que se han realizado en el mismo 0,56 determinaciones por vivienda, que frente al comparador obtenido de 1,20 nos conduce a un grado de control del orden del 47 por 100 del exigido por la Instrucción EH.

\subsection{Control realizado en edificios no residenciales}

En el Cuadro 3 se recogen datos relativos a la construcción de edificios en España obtenidos de AECC [1] y SEOPAN [3].

De los datos anteriores se deducen los reflejados en el Cuadro 4.

Puede observarse como la estructura tipo, teórica, para 20 viviendas de la figura 3 tiene una superficie media construida de $168 \mathrm{~m}^{2}$ por vivienda, que es del orden de la deducida a través de las estadísticas de construcción.

A partir de las viviendas terminadas (12.782) en Cantabria en el período 1984-88, aceptando las superficies medias construidas por vivienda terminada del Cuadro $4, y$ teniendo presentes las determinaciones de resistencia de hormigones en el quinquenio para cada tipo de edificio, puede llegarse a valorar el grado de control que se está llevando a cabo en cada uno de ellos, tal como se recoge en el Cuadro 5.

Se deduce del Cuadro 5 que el Control que se está llevando a cabo en edificios no residenciales es mayor que en los de viviendas, y en un grado que, habida cuenta de los valores deducidos para éstas (47 por ciento), tiende a las exigencias mínimas planteadas por la normativa vigente y puede estimarse en órdenes del 88 por ciento.

CUADRO 3. CONSTRUCCIÓN DE EDIFICIOS EN ESPAÑA EN EL PERIODO 1976-79

\begin{tabular}{|l|c|c|c|}
\hline AÑO & $\begin{array}{c}\text { N. }{ }^{\circ} \text { VIVIENDAS } \\
\text { TERMINADAS } \\
\text { (SEOPAN) }\end{array}$ & $\begin{array}{c}\text { M² CONSTRUIDOS } \\
\text { EN EDIFICACION } \\
\text { RESIDENCIAL }\end{array}$ & $\begin{array}{c}\text { M² CONSTRUIDOS EN }_{\text {EDIFICACIÓN NO }} \\
\text { RESIDENCIAL (Docente, } \\
\text { Sanitaria, Admtva., Industrial } \\
\text { y Deport.) }\end{array}$ \\
\hline 1976 & 319.825 & 55.030 .000 & 7.795 .000 \\
1977 & 324.384 & 53.370 .000 & 9.410 .000 \\
1978 & 318.870 & 47.445 .000 & 9.505 .000 \\
1979 & 260.774 & 44.600 .000 & 8.280 .000 \\
\hline Total años & 1.223 .853 & 200.445 .000 & 34.990 .000 \\
1976 a 1979 & & &
\end{tabular}




\section{CUADRO 4. VALORACIONES MEDIA EN EL PERIOODO 1976-79 EN EDIFICIOS PARA VIVIENDAS Y EN NO RESIDENCIALES}

\begin{tabular}{|l|c|c|}
\hline EDIFICACIÓN EN ESPAÑA (1976-79) & $\begin{array}{c}\text { EDIFICIOS } \\
\text { RESIDENCIALES }\end{array}$ & $\begin{array}{c}\text { EDIFICIOS NO } \\
\text { RESIDENCIALES }\end{array}$ \\
\hline $\begin{array}{l}\text { SUPERFICIE MEDIA CONSTRUIDA (M²) POR } \\
\text { VIVIENDA TERMINADA }\end{array}$ & $164 \mathrm{~m}^{2}$ & $28 \mathrm{~m}^{2}$ \\
$\begin{array}{l}\text { PORCENTAJE SOBRE EL TOTAL DE M² } \\
\text { CONSTRUIDOS EN ESPAÑA }\end{array}$ & $85 \%$ & $15 \%$ \\
\hline
\end{tabular}

\section{CUADRO 5. ESTIMACIÓN DEL GRADO DE CONTROL DE HORMIGÓN EN EDIFICIOS PARA VIVIENDAS Y EN NO RESIDENCIALES EN CANTABRIA (1984-88)}

\begin{tabular}{|l|c|c|c|}
\hline $\begin{array}{c}\text { EDIFICACIÓN EN } \\
\text { CANTABRIA (1984-88) }\end{array}$ & $\begin{array}{c}\text { ESTIMACIÓN DE } \\
\text { M² CONSTRUIDOS }^{2}\end{array}$ & $\begin{array}{c}\text { DETERMINACIONES } \\
\text { DE RESISTENCIA } \\
\text { (HORMIGÓN) }\end{array}$ & $\begin{array}{c}\text { N.0 DE } \\
\text { DETERMINACIONES } \\
\text { CADA 1.000 M }{ }^{2} \\
\text { CONSTR. }\end{array}$ \\
\hline DE VIVIENDAS & 2.096 .248 & 7122 & 3,4 \\
NO RESIDENCIAL & 357.896 & 2289 & 6,4 \\
\hline
\end{tabular}

\subsection{Evolución del control del hormigón}

En el Cuadro 6 se recoge la estimación que AECC [1] hizo del porcentaje de superficie de estructura de hormigón controlada en relación al total construido.

El Cuadro muestra que el Control de hormigón es cada vez mayor y presenta un incremento anual del orden del 5 por 100 . Según lo anterior, en el año 1986 (medio de nuestro estudio) se estaría realizando un control del orden del 55 por 100 de la superficie construida.
Veamos en qué medida se cumple lo anterior en nuestro caso. Teniendo presente el grado de control realizado en Cantabria en edificios de viviendas (47 por ciento) y en no residenciales ( 88 por 100 ) y aceptando el reparto del total de la superficie construida en la región según los valores del Cuadro 4 ( 85 por 100 en viviendas y 15 por ciento para el resto de edificios) se llega a un 53 por ciento de superficie controlada del total construido.

\section{CUADRO 6. PORCENTAJE DE ESTRUCTURAS DE HORMIGÓN CONTROLADAS EN ESPAÑA (1976-79)}

\begin{tabular}{|c|c|c|c|}
\hline AÑO & $\begin{array}{c}\text { M}^{2} \text { CONSTRUIDOS EN } \\
\text { TOTAL EN ESPAÑA }\end{array}$ & $\begin{array}{c}\text { ESTIMACION DE LOS M } \\
\text { CONTROLADOS (A.E.C.C.) }\end{array}$ & $\begin{array}{c}\% \text { DE } \\
\text { CONTROL }\end{array}$ \\
\hline 1976 & 62.825 .000 & 3.055 .000 & 5 \\
1977 & 62.780 .000 & 7.272 .000 & 12 \\
1978 & 56.950 .000 & 8.757 .000 & 15 \\
1979 & 51.880 .000 & 10.313 .000 & 19 \\
\hline
\end{tabular}




\section{CONTROL DE HORMIGÓN EN ESTRUCTURAS DE EDIFICACIÓN}

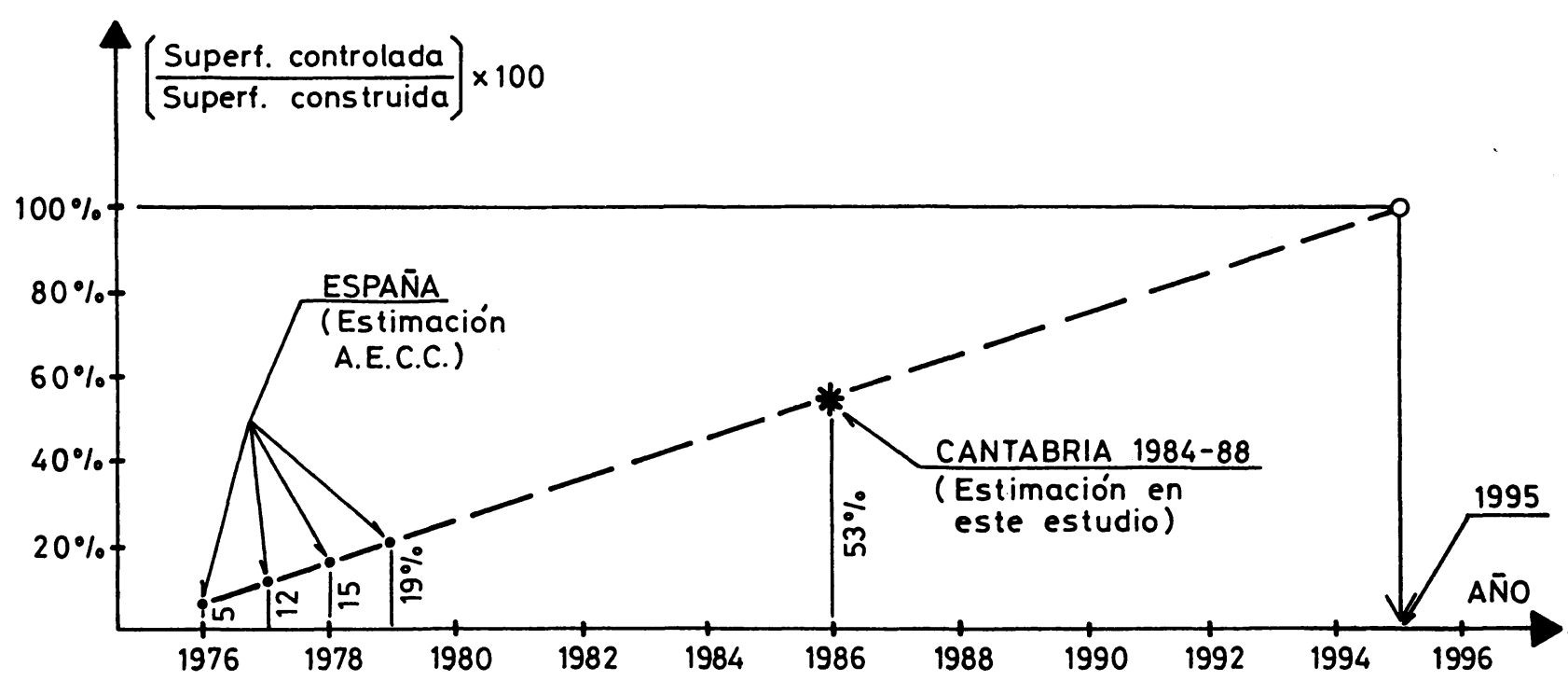

Fig. 4.-Estimación de la evolución del Control de hormigón en estructuras de edificación en España.

\section{VALORACIÓN DEL CONTROL DEL ACERO}

\subsection{Normativa}

Artículo 71, Control de calidad del acero, de EH-88. Para el caso de estructuras de edificación con control a nivel normal deben de realizarse en cada diámetro, y material, utilizando en la obra una serie de ensayos normalizados, los cuales se repetirán siempre que el consumo de acero de ese diámetro supere las $20 \mathrm{t}$.

\subsection{Control realizado en estructuras para viviendas}

Se considerará como estructura tipo del período la misma (fig. 3) que tomamos para el caso de hormigón y aceptaremos que en ella se han utilizado 6 diámetros diferentes de armaduras, como es frecuente.

En una obra de estas características el consumo de acero en cada diámetro no suele alcanzar las $20 \mathrm{t}$; por ejemplo la estructura tipo que nos ocupa lleva 63 t de acero, lo que nos conduce a tener que controlar 6 diámetros por obra.

Aceptando lo anterior, y teniendo presentes las viviendas terminadas en el quinquenio, llegamos a un total de 639 estructuras y de 3.834 controles necesarios en diferentes diámetros, que frente a los 571 realizados conducen a un grado de control del orden del 15 por ciento respecto al exigido por la normativa.
Se confirma, pues, la tendencia apuntada por el Cuadro 6 de incrementos de control del orden del 5 por ciento por año, según lo cual es previsible que, de mantenerse esta línea, hacia mediados de la década de los noventa (figura 4), se satisfagan las exigencias de control planteadas por la normativa.

\subsection{Control realizado en edificios no residenciales}

Con igual criterio que el utilizado en el caso de hormigón se llega al Cuadro 7.

Nuevamente se pone de manifiesto un mayor control en los edificios no residenciales frente a los de viviendas. Con los datos anteriores se deduce para aquéllos un grado de control del orden del 44 por 100 respecto al exigido por EH.

\subsection{Evolución del control del acero}

En la figura 5 se representa la evolución del número de controles del hormigón y del acero, a lo largo del quinquenio, en estructuras para viviendas. Ella pone de manifiesto para ambos materiales la tendencia de que el control que se realiza es cada vez mayor, como ya se mostró para el caso del hormigón. Dado el peso de las viviendas respecto al total de la construcción de edificios puede aceptarse lo apuntado como general para las estructuras de éstos. 


\section{CUADRO 7. ESTIMACIÓN DEL GRADO DE CONTROL DEL ACERO EN LAS ESTRUCTURAS DE EDIFICACIÓN (CANTABRIA, 1984-88)}

\begin{tabular}{|l|c|c|c|}
\hline $\begin{array}{c}\text { ESTIMACION EN } \\
\text { CANTABRIA (1984-88) }\end{array}$ & $\begin{array}{c}\text { ESTIMACION DE } \\
\mathrm{M}^{2} \text { CONSTRUIDOS }\end{array}$ & $\begin{array}{c}\varnothing \text { DE ARMADURAS } \\
\text { CONTROLADOS }\end{array}$ & $\begin{array}{c}\text { N. }{ }^{\circ} \text { DE DIAMETROS } \\
\text { CONTROLADOS CADA } \\
10.000 \mathrm{M}^{2} \text { CONST. }\end{array}$ \\
\hline DE VIVIENDAS & 2.096 .248 & 571 & 2,72 \\
NO RESIDENCIAL & 357.896 & 286 & 7,99 \\
\hline
\end{tabular}

\section{EVOLUCIÓN DEL CONTROL EN VIVIENDAS}

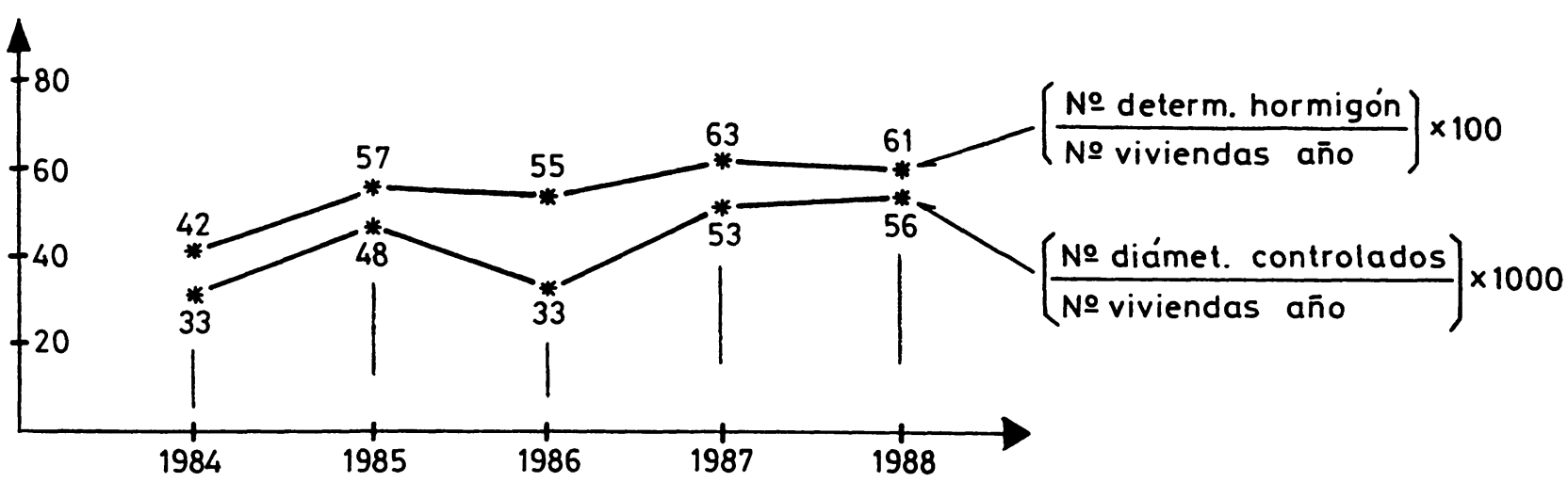

Fig. 5.-Evolución del control de materiales en estructuras para viviendas (Cantabria, 1984-88).

\section{CONCLUSIONES}

\subsection{En relación con el Control del Hormigón:}

- En estructuras para viviendas se está controlando del orden del 47 por 100 de las construidas, lo cual es insuficiente en relación con las especificaciones de la Instrucción EH que establece la obligatoriedad del control en su totalidad.

- En estructuras para edificios no residenciales el grado de control (88 por 100) está próximo al cumplimiento de los límites mínimos de la normativa.

- Respecto al total de las estructuras de edificación se están controlando del orden del 53 por 100 de las construidas y parece confirmarse que el control se incrementa anualmente del orden del 5 por 100, por lo que es previsible que hacia mediados de los años noventa se alcance el objetivo de la Instrucción.

\subsection{Respecto al Control del Acero:}

- El cumplimiento de la normativa vigente se hace en menor medida que lo expuesto para el hormigón. Se obtienen niveles de control del 15 por 100 en viviendas y del 44 por 100 en edificios no residenciales respecto al exigido por EH.

- Se manifiesta una tendencia hacia un mayor control del acero a lo largo del período estudiado.

\section{AGRADECIMIENTOS}

Deseo agradecer a los Organismos y personas que se citan seguidamente la colaboración prestada en la toma de datos que sustentan este estudio.

- EUROCONSULT, S. A. y a don Nicolás Laguna Martínez. 
- ICINSA, S. A. y a don José Manuel Bonachea Martínez.

- LABORATORIO DE CALIDAD DE LA EDIFICACIÓN DE LA DIPUTACIÓN REGIONAL DE CANTABRIA y a don Angel Pérez Cruz.

- HONGOMAR, S. A. (Hormigones Montañeses) y a don Jacinto López Lavid.

\section{REFERENCIAS}

[1] A.E.C.C. (Asociación Española para el Control de la Calidad). Encuestas sobre control de calidad en la
Edificación. Primer Informe, Noviembre 1981.

[2] MINISTERIO DE OBRAS PUBLICAS Y URBANISMO. Instrucción EH-88 para el Proyecto y la Ejecución de Obras de Hormigón en Masa o Armado.

[3] SEOPAN (Asociación de Empresas Constructoras de ámbito nacional). Informes anuales sobre la Construcción.

[4] VILLEGAS, L. Una valoración sobre la Calidad de los Proyectos y la Ejecución de las obras de Edificación en Cantabria/España. Informes de la Construcción n. 366 (1984).

\section{publicaciones del ICCET/CSIC}

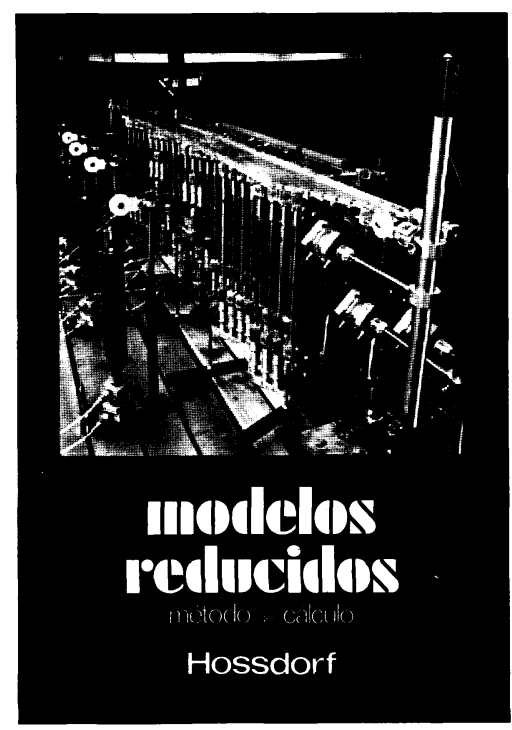

Modelos reducidos. Método de cálculo

H. Hossdorf, Ingeniero Civil

La técnica de los ensayos en modelos reducidos de estructuras sufre hoy dia una decisiva metamorfosis. Hasta hace poco era un medio más bien de artesania, que no siempre era tomado en serio por los académicos teorizantes para comprender el comportamiento resistente de las estructuras complejas y al que se acudió las más de las veces, como a un último remedio debido a sus indiscutibles insuficiencias. Sin embargo, en poco tiempo y gracias a su conexión con los ordenadores digitales, se ha transformado en un instrumento cientificamente valioso, que no puede quedar a un lado en la práctica diaria del Ingeniero Proyectista.

Un volumen encuadernado en cartoné plastificado con lomo de tela, de $17 \times 24 \mathrm{~cm}$, compuesto de 250 páginas, 158 figuras y fotografias.

Precios: 1.800 ptas.; \$ USA 26.00 .

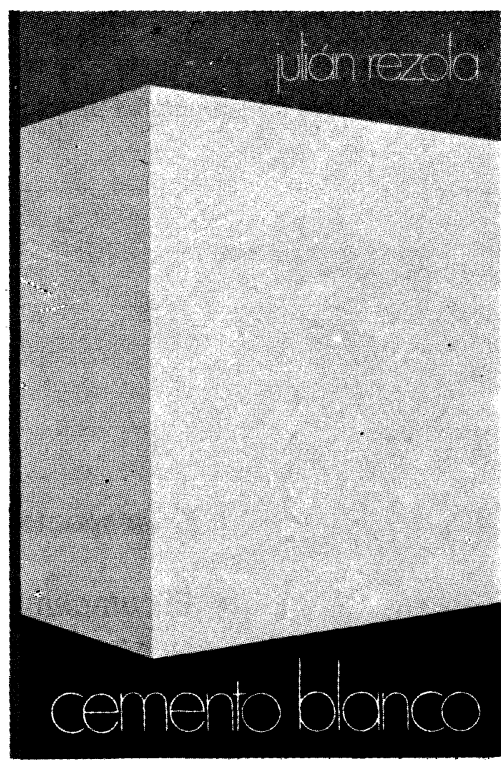

Cemento blanco

Julián Rezola

Ingeniero Químico Dipl. I. O. S.

Sabido es que existe una extensa y documentada bibliografía sobre el cemento gris: en cambio, no puede decirse lo mismo acerca del cemento portland blanco, ya que los escritos existentes se refieren tan sólo a algunas peculiaridades que le distinguen de aquél.

El autor nos ofrece sus profundos conocimientos y su larga experiencia tanto en laboratorio como en fabricación.

La parte descriptiva del libro se complementa con gráficos, diagramas y fotografias de gran utilidad, destinados a conseguir la aplicación apropiada de Un volumen encuadernado en cartoné policerado, de
$17,4 \times 24,3 \mathrm{~cm}$, compuesto de 395 páginas, numerosas figuras, tablas $y$ ábacos.

Precios: España, 1.700 ptas.; extranjero, \$ 24.

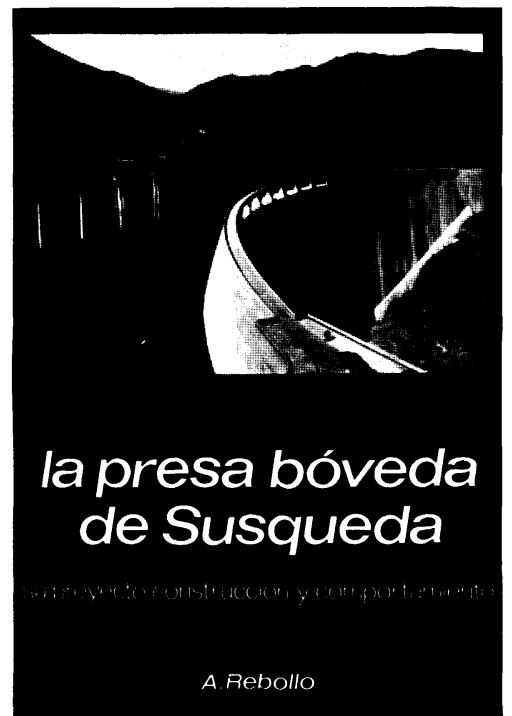

La presa bóveda de Susqueda

A. Rebollo,

Dr. Ingeniero de Caminos

El esfuerzo del constructor de presas se sitúa, por su pretensión de perennidad, a contracorriente de las tendencias de la civilización actual, caracterizada por lo fungible. Pueden evocarse las grandes. presas en funcionamiento o en construcción que están envejeciendo $y$ reclaman los cuidados gerontológicos para mantener y perfeccionar su perennidad. En la suliena pretension de peras, grandes 0 pequeñas, son poras nuevas riesgos ecológicos $y$ a veces, catostóficos, de aumentan con el envejecimiento la gerolicos, que las presas es todo un ecmpazo. La accion adola de le Arturo Rebollo en este terreno marca un camino a seguir para a seguir para todos los que aman su propia obra con

Un volumen encuadernado en cartone plastificado con lomo de 408 púginas, 330 figuras $y$ fotografias $y 39$ tablas.

Precios: 1.700 ptas.; extranjero, \$ USA 24.00. 